\title{
Automated incoming control with advanced functionality for processing frozen meat raw materials on continuous process lines with application of single-stage grinding
}

\author{
Boris Kapovsky*, Tatyana Kuznetsova, and Anton Lazarev \\ V.M. Gorbatov Federal Research Center for Food Systems of Russian Academy of Sciences, 109316, \\ 26, Talalihina Street, Moscow, Russian Federation
}

\begin{abstract}
The article presents the functionality of the incoming control system (ICS) of raw materials (frozen blocks) processed on an automated line for production of meat products. The parameters of the cutting process of raw materials depending on its temperature and the effectiveness of procedures for recognizing foreign inclusions in the raw materials have been studied. It has been determined that the key controlled thermodynamic parameters for a frozen block are not only the temperature of the meat layer directly in the grinding zone, but also the temperature of the subsequent layers (a mathematical model of the temperature front propagation) that determine the change in the plastic properties of the grinded meat. The authors performed a mathematical calculation of heat transfer in a block of frozen meat in the process of its grinding and an approximate method for calculating the assessment of the heat of phase transition is offered. A cost-effective option was found (based on a neural network-classifier that considers the cost function) for detecting foreign inclusions in raw materials (when creating an ICS), which makes it possible to determine the block size that can be triggered without the risk of entering the specified inclusion into the grinding product.
\end{abstract}

\section{Introduction}

In the domestic practice of producing meat products from frozen block meat, as a rule, continuous control of blocks using manual labor with preliminary defrosting of raw materials is used. This does not allow to fully automate the process of the production of meat products during their in-line production. Use of foreign equipment for incoming control of raw materials at medium and small meat processing enterprises is limited due to its high cost. These factors determine the relevance of development of domestic-made technical means for automated control of frozen meat raw materials, including those with expanded functionality in the case of its application on continuous processing lines using one-stage grinding of frozen meat. Use of an innovative method of one-stage grinding of raw materials frozen in the form of blocks involves use of an intelligent control system

\footnotetext{
*Corresponding author: b.kapovski@fncps.ru
} 
(IMS) of the process in the conditions of functioning the automated line for production of meat products. The incoming control system (ICS) of raw materials processed on an automated line is a subsystem of the IMS, which also includes predicting the characteristic size of the resulting meat chips based on a mathematical model of the raw material cutting process with a multi-blade tool [1], and controlling the mixing of grinded meat with ingredients in a minced meat mixer for production of grinded meat with optimal functional and technological characteristics.

To detect foreign objects in meat raw materials, radiation in the X-ray range of the spectrum is used. The corresponding technical systems do not provide for determination of the coordinates of the foreign inclusion. For the purpose of its further localization, the entire object is rejected, for example, a block of frozen meat raw materials. However, with a one-stage grinding of frozen block meat in the conditions of an automated line, it is advisable not only to obtain the indicated coordinates in an explicit (digital) form relative to a given coordinate system, but also - using these coordinates, considering the sequential actuation of a block of meat with a single-stage grinder, - to partially process the raw material to the location foreign inclusion.

In the applied industrial incoming control systems, the combination of the option of monitoring the temperature regime of raw materials with the option of detecting foreign inclusions in it is not provided. Temperature control is carried out at the subsequent stages of raw material processing, after incoming control. When designing the ICS for automated lines of in-line production of meat products using one-stage grinding, one shall consider the features of the process of cutting frozen raw materials with a multi-blade tool. Experimental study has proven that the temperature of meat in the cutting zone (the area of contact of the cutter teeth with the cutting surface of the meat block) is close to the cryoscopic temperature of frozen raw materials. In this temperature range, the plastic properties of frozen meat are manifested. When the raw material is fed to the cutter, part of it can be pushed into the space of the groove between the cutter teeth, as a result of which the thickness of the cut meat chips will be greater than the specified value (Fig. 1). The variance in the size of the meat chips increases, which leads to a decrease in the quality of the meat product. To reduce the dispersion of the chip size due to the manifestation of the plastic properties of meat, the frequency of rotation of the cutting tool shall be increased when the raw material is heated during the grinding process. Maintaining the maximum speed of rotation of the cutter, close to the synchronous speed of rotation of the drive electric motor, during the entire time period of grinding a block of meat is impractical in order to reduce energy consumption for the process of cutting raw materials.

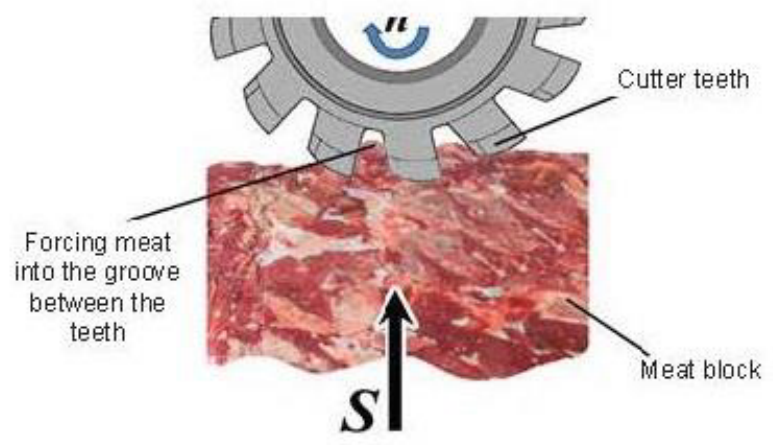

Fig. 1. Manifestation of the plastic properties of meat ( $\mathrm{n}$ is the rotational speed of the cutter; $\mathrm{S}$ is the supply of a block of raw materials to the cutting tool). 
Hence, the problem of regulating the operating parameters of the cutting process of raw materials, depending on its temperature arises. Therefore, it is necessary to measure the temperature of meat during grinding in real time of the operation of a single-stage grinder. The authors [2] offer a method for temperature control of raw materials in the process of its processing using one-stage grinding. However, the question remains: how many ranges of temperature of the surface layer of meat is advisable to use as triggers for switching the operating parameters of the cutting process of raw materials. The boundaries of these temperature ranges are determined rather conditionally on the basis of an experimental study of one-stage grinding the frozen raw materials, and can be movable when the experimental conditions change. In this case, it is advisable to increase the number of boundary temperature values, that is, to reduce the extrapolation step. This will make it possible to more accurately respond to changes in the plastic properties of frozen meat with an increase in its temperature. In the source [2], as a reference, it is offered to take the measured (for example, a thermocouple) value of the temperature of the surface layer of the meat of a frozen block, which is crushed last from a batch of blocks delivered from the freezer. The peculiarity of grinding the frozen meat by the milling method shall include the rigid fastening the raw material block in the guide planes of the working chamber of the grinder. This is necessary to obtain meat chips of a given size, due to the applied technology of further processing the homogeneous minced meat. Arrangement of the processing conditions for meat blocks in this sense fully corresponds to the conditions for fastening parts in the metalworking industry during turning and milling. Experimental grinding of meat blocks on an experimental installation of one-stage grinding of the design of the Federal State Budgetary Scientific Institution Federal Research Center of Food Systems named after V.M. Gorbatov, RAS, showed deformations of the surface layer of block meat due to the pressure of the guide planes. It is known that plastic deformation of a material leads to its heating [3]. In addition, when a rigidly fixed block of meat moves in a box of guiding planes of the working chamber of the grinder, the work of friction forces is converted into heat energy, which heats the surface layer of meat. Within experimental grinding on the experimental setup, partial defrosting the surface layers of meat with release of moisture was observed. Therefore, the temperature regime of the surface layer of meat adjacent to the zone of contact with the multi-blade tool in the working chamber of the grinder may differ significantly from the conditions that determine the temperature regime of the same layer in the block outside the grinder prior to grinding, for example, in a floor cart. Hence follows the task of determining the temperature of the meat layer directly in the grinding zone to correctly consider the changes in its plastic properties during heating.

The offered concept of the ICS allows solving the tasks formulated above.

\section{Methods}

Selection of the method for calculating heat transfer in a block of frozen meat during its grinding was carried out in accordance with modern concepts of heat transfer processes in composite materials of heterogeneous structure [3].

Selection of a method for recognizing foreign inclusions in the volume of a frozen block of meat according to full or partial specification was carried out in accordance with provisions of the modern theory of sample recognition $[4,7]$. 


\section{Results}

Exact solutions of the equations of thermal conductivity when heating a frozen block of meat during its processing is difficult to apply in engineering practice under production conditions. Hence, it becomes necessary to obtain approximate solutions of these equations, which are acceptable in accuracy for use in meat processing. For this, the method of averaging the time derivative included in the heat conduction equation is used. The method is based on the hypothesis of presence of a temperature front propagating from the surface layers of the heated body to the central layers with a finite rate due to the relaxation time. For meat products, the relaxation time, depending on the structural and mechanical properties, varies within $10^{0}-10^{1} \mathrm{~s}$, which leads to a heat propagation rate of $10^{0}-10$ ${ }^{1} \mathrm{~m} / \mathrm{h}$ [8]. The temperature front is understood as the interface between the regions of the object that are perturbed and not perturbed by the thermal effect. In this case, the object is understood as meat chips in the form of a plate when it is separated from the frozen block of meat during the grinding process. The following assumptions are introduced: the temperature front moves symmetrically relative to the geometric center of the body; all points lying ahead of the front have a temperature different from the initial one, while the temperature behind the front and at the front itself is considered equal to the initial one [8]. Introduction of the temperature front as a mathematical model of heat transfer assumes the final rate of heat propagation in the body, in particular, in the meat product.

In the process of moving the temperature front, two phases are considered: 1) the first phase in time covers the period during which the temperature front passes the distance from the boundary plane of the body to its geometric center; 2) the second phase characterizes the change in temperature in the center of the body over time. In the case of calculating the temperature in the meat chips obtained during one-stage grinding of a frozen meat block, only the first phase of the temperature front advance is of practical interest, that is, the transfer of heat from the source (cutting tool blade) to the boundary regions of the meat chip plate, and then, towards its geometric center. The second phase is not considered, since after separation of the meat chips from the block mass, it is almost instantly removed from the cutting zone. At the next stage of processing the crushed raw materials, the temperature of the meat chips is measured by means of an ISU technological process on an automated line in order to optimally mix the minced meat in a minced meat mixer.

The mathematical model of the propagation of a temperature front in meat chips is applicable for an approximate solution of the Fourier equation using the method of averaging the time derivative. A one-dimensional problem of heating meat chips from the boundary region of contact with the cutting blade to its geometric center (middle of the thickness) is considered. The heat conduction equation will have the form [8]:

$$
\frac{1}{\mathrm{a}} \frac{\partial \mathrm{T}}{\partial \tau}=\frac{\partial^{2} \mathrm{~T}}{\partial \mathrm{x}^{2}}+\frac{v}{\mathrm{x}} \frac{\partial \mathrm{T}}{\partial \mathrm{x}}
$$

where $\mathrm{T}-$ chip temperature; $\mathrm{a}-$ thermal diffusivity; $\tau-$ time; $\mathrm{x}-$ current coordinate; $v$ - body shape coefficient, for plate $v=0$.

To solve equation (1), we set the boundary conditions. The boundary condition of the first kind will be as follows:

$$
\mathrm{T}(\mathrm{x} ; 0)=\mathrm{T}_{\mathrm{b}}=\text { const },
$$

This condition means that the initial temperature at the edge of the meat chips at the initial moment of time is constant. Let us also specify that:

$$
\left(\frac{\partial T}{\partial \mathrm{x}}\right)_{\mathrm{x}=0}=0
$$


This condition means that the temperature along the temperature front line is unchanged. This condition seems to be justified due to the small length of the meat chips. Note that the mathematical expectation of the length of the meat chips when grinding block meat with a cutter with a fine tooth in accordance with GOST 29092-91 is 166.64 microns [3].

To describe the heat exchange of meat chips with the environment, a boundary condition of the third kind shall be set:

$$
\left[\frac{\partial \mathrm{T}}{\partial \mathrm{x}}-\frac{\alpha}{\lambda}\left(\mathrm{T}_{\mathrm{c}}-\mathrm{T}\right)\right]_{\mathrm{x}=1}=0
$$

Where: $\alpha-$ heat transfer coefficient; $\lambda-$ thermal conductivity coefficient of frozen meat; $\mathrm{l}$ - the characteristic size of the meat chips (half of its thickness).

Note that the axis " $\mathrm{x}$ " is oriented perpendicular to the lateral edges of the meat chips, regarded as a plane-parallel plate (Fig. 2).

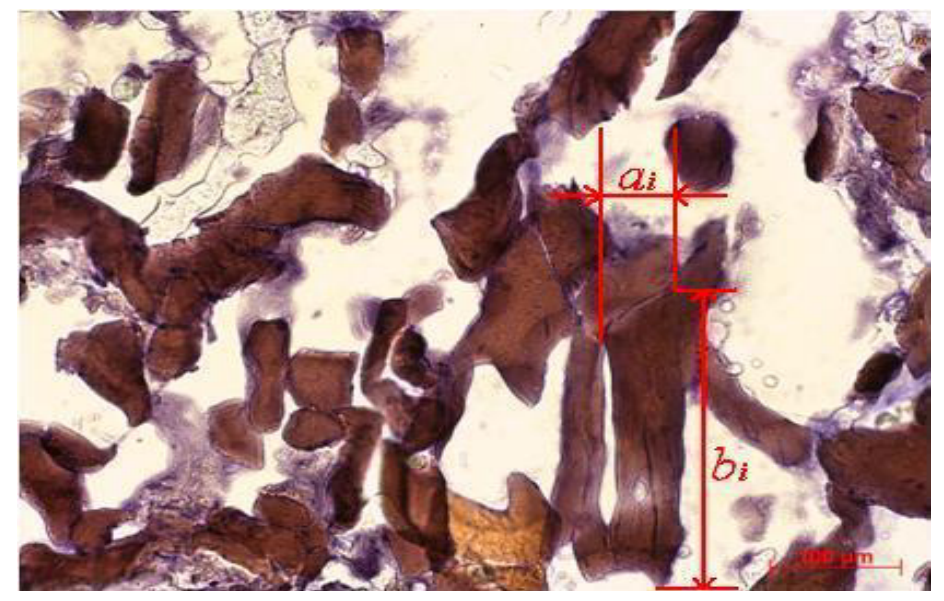

Fig. 2. Sizes of meat chips (beef): $a_{i}-$ chip thickness, microns; $b_{i}-$ chip width, microns.

Let's introduce dimensionless complexes:

$$
\begin{gathered}
\mathrm{T}_{1}=\frac{\mathrm{T}-\mathrm{T}_{b}}{\mathrm{~T}_{\mathrm{c}}-\mathrm{T}_{b}}, \\
\frac{\mathrm{x}}{\mathrm{l}}=\xi, \\
\frac{\mathrm{a} \tau}{\mathrm{l}^{2}}=\mathrm{Fo}-\text { the Fourier criterion, }
\end{gathered}
$$

Then equation (1) in dimensionless form can be written as follows [8]:

$$
\frac{\partial \mathrm{T}_{1}}{\partial \mathrm{Fo}}=\frac{\partial^{2} \mathrm{~T}_{1}}{\partial \xi^{2}}+\frac{v}{\xi} \frac{\partial \mathrm{T}_{1}}{\partial \xi}
$$

According to the averaging method, the left side of equation (8) is replaced by the function [8]: 


$$
\varphi_{\nu}^{(\beta)}=\frac{1}{2[1+(\beta-2)] \psi^{v+1}} \int_{(2-\beta) \psi}^{1} \frac{\partial \mathrm{T}_{1 v}^{(\beta)}}{\partial \mathrm{Fo}} \xi^{v} \mathrm{~d} \xi,
$$

where $\beta$ - index of the corresponding heating phase; $\psi=\psi\left(\mathrm{Fo}_{0}\right)-$ thermal disturbance boundary equation for the first heating phase.

Considering (9), equation (8) is replaced by an approximate equation:

$$
\frac{\partial^{2} \mathrm{~T}_{1 v}^{(\beta)}}{\partial \xi^{2}}+\frac{v}{\xi} \frac{\partial \mathrm{T}_{1 v}^{(\beta)}}{\partial \xi}=2(1+v) \varphi_{v}^{(\beta)}(\mathrm{Fo}),
$$

Since the heated meat chips are considered as a plate, and the first phase of its heating is also calculated, then $\nu=0, \beta=1$.. Then equation (10) has the form:

$$
\frac{\partial^{2} \mathrm{~T}_{10}^{(1)}}{\partial \xi^{2}}=2 \varphi_{0}^{(1)}(\mathrm{Fo})
$$

Function $\varphi_{0}^{(1)}(\mathrm{Fo})$, depending on the Fourier criterion, is obtained from the averaging condition [8]:

$$
2 \varphi_{0}^{(1)}(F o)=\frac{1}{1-\psi} \int_{\psi}^{1} \frac{\partial T_{10}^{(1)}}{\partial F o} d \xi
$$

By successively integrating (11) with a changing initial condition (2), it is possible to determine the desired temperature of the chips, which can be used to judge the change in the plastic properties of the grinded meat.

To set the boundary condition of the first kind (2) in solving the heat conduction equation, it is necessary to experimentally measure the initial temperature in the contact zone of the cutting tool blade with the raw material at the initial time of separation of the meat chips from the block mass. Technically, it is possible to do this in a non-contact way at point 1 of the entry of the tool tooth into the mass of the meat block (Fig. 3).

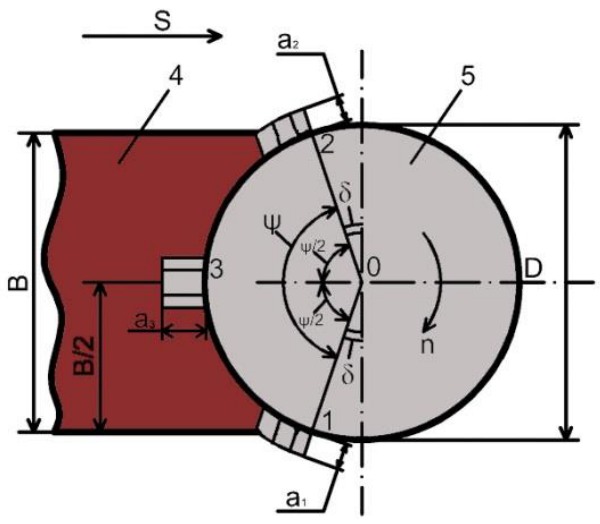

Fig. 3. Milling diagram of a frozen block of meat ( $\mathrm{S}$ - block feed to the cutter; $\mathrm{n}$ - cutter rotation frequency; a1, a2, a3 - thickness of meat chips at points 1, 2, 3; B - cutting width; D - cutter diameter; $\psi$-contact angle; $\delta$ - angle on the cutting path at points 1,$2 ; 4$ - block of frozen meat; 5 - cutter). 
Then, by calculation, the average temperature along the cutting arc $(1-3$, Fig. 3$)$ is determined under the assumption that in the center of the block (point 3, Fig. 3) the initial temperature is unchanged and equal to the storage temperature of the raw material in the freezer before grinding.

The method of non-contact temperature measurement in the manufacturing industry is registration of thermal radiation from individual parts of a part or tool during processing [9]. The diagram of the non-contact temperature measurement in the cutting zone of the grinder of frozen block meat is shown in Fig. 4.

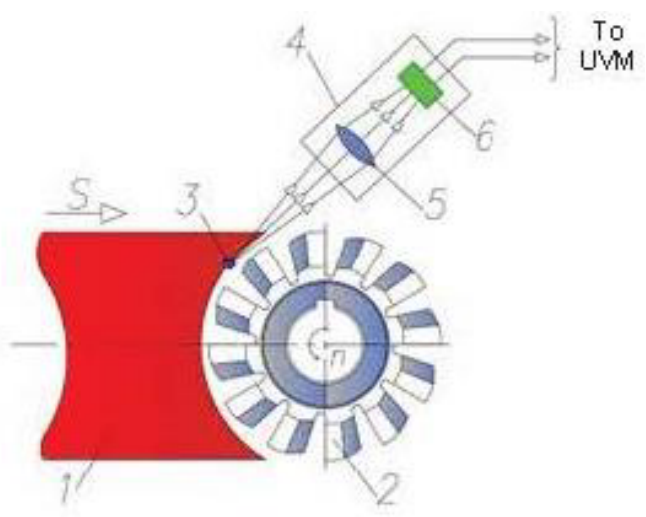

Fig. 4. Boundary layer temperature measurement diagram ( 1 - frozen meat block; 2 - grinder milling shaft; 3 - area on the cutting surface of the meat block; 4 - measuring device; 5 - lens; 6 - IR radiation sensor; $\mathrm{S}$ - feed direction of the raw material block to milling shaft of the grinder; $n$ is the rotational speed of the milling shaft of the grinder; UVM is a control computer (industrial computer), a component of the ISU).

When crushing the block of frozen meat 1 with the milling shaft of the grinder 2 , the thermal radiation of the contact area of the raw material with the multi-blade tool 3 is accumulated in the lens 5 and transferred to the photosensitive element 6 . The signal is amplified in the electronic amplifier (not shown in Fig. 4) of the measuring device 4 and is ultimately transmitted to the UVM. The reference indicates [9] that a signal in the IR range can be received from the contact area of $0.04 \times 0.07 \mathrm{~mm}$ with the measurement accuracy of $\pm 2-3 \%$.

Obviously, the temperature on the surface of the meat layer, measured experimentally, differs from the temperature in the center of the same meat layer, that is, in the center of the meat block. In the practice of refrigeration technology, there are approximations of the average volumetric temperature of bodies of a simple shape under the assumption of a parabolic temperature distribution over the cross section of the body [10]. In this case, the section of the body shall be understood as the cutting surface of the frozen meat block at the current time. The cutting surface of the meat block is represented as a plane-parallel figure in the form of a parallelepiped section - the shape of the meat block in accordance with GOST R 54704-2011.

We will place the origin of the coordinate system in the center of the meat block, and denote the temperature in the center of the block $\mathrm{T}_{\mathrm{c}}$. Axis " $\mathrm{x}$ " will be directed along the normal to the block surface, the temperature of which we denote as $\mathrm{T}_{\mathrm{s}}$. The distance from the center of the meat block to its surface will be denoted by $0.5 \mathrm{~N}$, where $\mathrm{H}$ is the height of the block of industrial standard size. Note that the block height $\mathrm{H}$ corresponds to the milling width B (Fig. 3) with a horizontal cutting shaft. Then, in accordance with the accepted assumption about the parabolic temperature distribution over the block section, the 
temperature of the point on the cutting surface of the meat block with the coordinate "x" can be represented as:

$$
\mathrm{T}_{\mathrm{x}}=\mathrm{T}_{\mathrm{s}}-\left(\mathrm{T}_{\mathrm{x}}-\mathrm{T}_{\mathrm{c}}\right) \frac{\mathrm{x}^{2}}{(0,5 \mathrm{H})^{2}},
$$

where $\mathrm{T}_{\mathrm{x}}$ - temperature at the point " $\mathrm{x}$ ".

Solving equation (13) for a set of points in the interval $\mathrm{x}_{\mathrm{c}}<x<\mathrm{x}_{s}$, where $\mathrm{x}_{\mathrm{c}}=0, \mathrm{x}_{\mathrm{s}}=$ $0,5 \mathrm{H}$, constitute a set of boundary conditions of the first kind for an approximate solution of the heat conduction equation (11).

When frozen meat is heated in the zone of its contact with the blades of the grinder's working tool during cutting, a phase transition "ice - water" or, in the classical formulation, "solid - liquid" occurs. Presence of such a transition is a positive factor in the technology of one-stage grinding of frozen meat, since a kind of "thermal barrier" is created that prevents overheating of the raw material during the grinding process. It shall be noted that microstructural studies of grinded meat obtained on an experimental unit for one-stage grinding of the design of the Federal State Budgetary Scientific Institution Federal Research Center of Food Systems named after V.M. Gorbatov, RAS, did not record cases of thermal coagulation of meat proteins when it was ground with cutters of different designs at all cutting modes.

An approximate calculation method can be offered to estimate the heat of phase transition. The IMS with a technological process on an automated line is equipped with equipment for monitoring the chemical composition of crushed raw materials. For express analysis of the chemical composition in real time, it is advisable to use a unit similar to ProFoss. This unit performs direct analysis of the sample moving in the process stream. The principle of operation of the device is based on reflected IR spectroscopy. The scanning time is $5-50 \mathrm{~ms}$, the result is issued every 3-15 seconds. Calculations show that grinding of a block of frozen meat of industrial standard sizes with a milling grinder occurs within 2023 seconds. In view of the fact that the ICS is intended for an automated line of in-line production of meat products, the information obtained on the chemical composition of grinded meat can be accumulated and generalized to a batch of blocks of frozen raw materials. Based on the data obtained by measurements in automatic mode, it is possible to estimate the amount of free moisture per unit mass of crushed raw materials. Obviously, this moisture can include condensate of water vapor contained in the environment; ice crystals that have passed into a different state of aggregation due to mutual friction in the flow of crushed raw materials or heating in the environment, and etc. However, it can be assumed that a significant part of the free moisture was formed as a result of the thermal effect on the ice crystals contained in the frozen raw material, heated during the grinding of the cutting edges of the cutting tool blades of the grinder. Therefore, indirectly, with a certain measurement error, the reasons for which were named above, it is possible to estimate the amount of moisture formed as a result of the "ice-water" phase transition during the production of meat chips. According to the well-known thermodynamic dependences [3], it is possible to determine the amount of heat required for this and refer it to the unit mass of meat chips obtained over the same time period. In a first approximation, this will be an estimate of the amount of heat spent on the phase transformation of ice into water and, as a result, a decrease in the heat load on the meat chips. This reduction in heat load can be taken into account by a correction factor when calculating the temperature of the meat chips in the first phase of their heating.

Development of a technical system for the classification of images of foreign inclusions in frozen raw materials intended for processing on an automated line is reduced to the sequential solution of the following problems $[5,6]$ :

- detector development; 
- the task of generating features is the selection of those features that describe the images of foreign inclusions with sufficient completeness;

- the task of feature selection is the selection of the most informative features for classification;

- the task of constructing a classifier is the synthesis of a decision rule, according to which, on the basis of a vector of features, an object is assigned to a particular class.

A neural classifier network can be built in two ways: 1) the network architecture is varied to accurately classify use cases; 2) synoptic weights and thresholds are selected for the given architecture of the classifier network. To build a neural network-classifier of foreign inclusions in meat raw materials processed on an automated line and designed according to the second method, a criterion is introduced in the form of a cost function that must be minimized [7].

Fig. 5 shows the diagram of the ICS operation for detecting foreign inclusions in the block of frozen raw materials. When the detector detects a foreign object in the block of raw materials, the UVM determines the size of the block, which can be triggered without the risk of getting into the grinding product of the specified inclusion. A layer of meat containing a foreign object (part of a block of raw materials with foreign inclusions - Fig. 5) cannot be crushed and has dimensions regulated by the relevant process documentation.

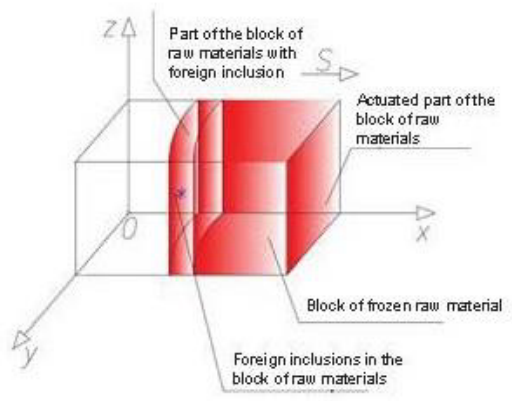

Fig. 5. Diagram of the ICS operation for detecting foreign inclusion in the block of frozen raw materials.

Substandard raw materials are removed automatically from the feeding conveyor of the automated line. Further, in order to localize foreign inclusion, the specified layer of meat is removed, for example, with a hand cutting tool (saw), counting the layer thickness from the characteristic concave cutting surface (Fig. 6) left by the cutting shaft of the grinder on the block of raw materials, in accordance with the specified size of this thickness specified in the process documentation.

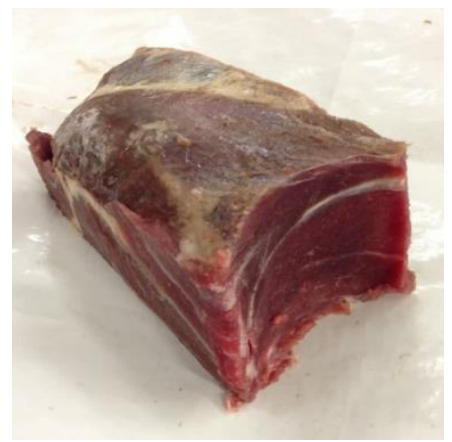

Fig. 6. Cutting surface of frozen meat block during its one-stage grinding. 


\section{Discussion}

Based on the studies carried out, we will formulate an algorithm for the operation of the ICS to determine the thermodynamic parameters in the boundary layer of meat of the frozen block of raw materials during its one-stage grinding:

- by the method of non-contact measurement, the temperature in the zone of entry of the blades of the working tool of a one-stage grinder into the surface layer of the frozen meat block is determined. The temperature in the center of the meat block is taken equal to the storage temperature of raw materials in the freezer before grinding.

- With the assumption of a parabolic temperature distribution over the section of the meat block, the initial temperature values of the raw material are determined for each layer of meat removed by the blades during the sequential actuation of the block in the process of its advancement in the working chamber of the grinder. The indicated temperature values are taken as boundary conditions of the first kind for solving the heat transfer equation in meat chips along the cutting arc.

- According to the obtained set of approximate solutions of the heat transfer equation in meat chips along the cutting arc, the average temperature in the meat chips along the cutting surface at the initial time of separation of the meat chips from the block is calculated.

- According to the temperature values calculated in this way for each layer of meat separated from the block mass when it is sequentially triggered, with a certain extrapolation step, the temperature is determined at which the operating parameters of the cutting process shall be changed to maintain a given degree of raw material grinding.

It is also possible to formulate the principles of constructing the ICS for detecting foreign inclusions in raw materials:

- $\quad$ as a detector, a source operating in the region of the spectrum corresponding to $\mathrm{X}$ ray radiation shall be used.

- to generate the features of the recognized object (foreign object in the raw material), use the texture of the object image (gradation of shades of gray in the monochrome image of the object), descriptive features (figure perimeter, figure area, figure non-circularity, bending energy) to describe the object shape.

- the features of an object shall be selectively selected in order to reduce computational complexity. Before selection, feature vectors are processed (outliers are removed, features are normalized).

- $\quad$ to build a classifier, a multilayer neural network with a given architecture is used. The function of synoptic weights and thresholds is introduced as a criterion for operation of the classifier network, for which the optimization problem shall be solved.

- In order to further localize foreign inclusion, increase the productivity of the automated line, economical consumption of raw materials, the ICS forms a command for partial grinding of the block of frozen raw materials to the location of the foreign object.

\section{Conclusion}

In the offered algorithm for the ICS operation for calculating the thermodynamic parameters of the boundary layer of meat in order to regulate the grinding mode of raw materials, one can see the principles used in modern tracking automatic control systems. In this case, a response to small temperature changes that do not cause significant changes in the plastic properties of meat is not expected. However, in contrast to the two-zone temperature control considered in the source [2], where only one boundary temperature value was noted (minus $12^{\circ} \mathrm{C}$, at which about $86 \%$ of the total amount of free moisture in 
meat is frozen), in the offered method of temperature control, the boundaries of the ranges of changes in the temperature of meat during grinding can be determined experimentally and by calculation through a given extrapolation step during layer-by-layer processing of the block of raw materials on the automated meat production line products. In addition, the offered method for temperature control of raw materials considers the heating of meat during the transition to the heat of work of friction forces during the movement of the block in the guide planes of the working chamber of the grinder.

For the synthesis of the ICS classifier, the multilayer neural network has been offered in connection with the significant advantages that have led to its widespread use in technical applications. The advantages of neural networks include the fact that they take up a small amount of memory, since only the structure of the neural network is preserved, and they provide a high speed of operations. In the case under consideration, this is important, since a significant amount of parallel computational work is envisaged for the UVM, which completes the ICS, to calculate the thermodynamic parameters of the boundary layer of meat. Decrease in the required memory volume and the high speed of operations will reduce the material costs for the technical equipment of the ICS project, which will increase its commercial attractiveness.

\section{References}

1. V.I. Ivashov, B.R. Kapovsky, P.I. Plyasheshnik, V.I. Pchelkina, E.L. Iskakova, Chemistry and technology, 5, 48 (2018)

2. A.B. Lisitsyn, A.N. Zakharov, D.A. Maximov, B.R. Kapovsky, V.G. Zhukov, Pat. 2529172, Russian Federation, Automatic control system for grinding food products frozen in blocks (2013)

3. A.A. Samarsky, Computational Heat Transfer (Moscow, Editorial URSS, 2003)

4. S. Khaikin, Neural networks (Moscow, Williams Publishing House, 2006)

5. A. Subramanian, S. Chitlangia, V. Baths, Neural Networks (to be published, 2021)

6. Y. Zhang, F.C. Morabito, D. Shen, K. Muhammad, Neural Networks, 133, 101 (2021)

7. L. Mestetskiy, Mathematical Methods for Pattern Recognition (Moscow, Moscow State University, 2002)

8. A.M. Brazhnikov, Analytical Methods for Studying the Processes of Heat Treatment of Meat Products (Moscow, Food Industry, 1974)

9. P.I. Yasheritsyn, Cutting Theory (Minsk, New Knowledge, 2006)

10. G.B. Chizhov, Thermophysical Processes in Refrigeration Technology of Food Products (Moscow, Food Industry, 1979) 